\title{
Multicolor symbology for remotely scannable 2D barcodes
}

\author{
Alexander D. Wissner-Gross ${ }^{\mathrm{a}}$, Timothy M. Sullivan ${ }^{\mathrm{b}}$ \\ ${ }^{a}$ Dept. of Physics, Harvard University, 20 Prescott Street, Cambridge, MA 02138 USA; \\ ${ }^{b}$ Dept. of the History of Art, Yale University, P.O. Box 1952, Brookline, MA 02446 USA
}

\begin{abstract}
There has been much recent interest in mobile systems for augmented reality. However, existing visual tagging solutions are not robust at the low resolutions typical of current camera phones or at the low solid angles needed for "across-the-room" reality augmentation. In this paper, we propose a new 2D barcode symbology that uses multiple colors in order to address these challenges. We present preliminary results, showing the detection of example barcodes in this scheme over a range of angles.
\end{abstract}

Keywords: 2D barcode, multicolor, optical tag, symbology, augmented reality, cameraphone

\section{INTRODUCTION}

There has been much recent interest in mobile systems for augmented reality. Existing systems are either tag-based or tag-less. Tag-less systems typically rely on pattern recognition of known objects in images, and have the advantage of not requiring new tag hardware. However, tag-less systems have the security disadvantage of ambiguous user ownership of those image objects. In contrast, tag-based systems allow explicit ownership of different augmentations. Passive tag-based systems may be grouped into optically visible (e.g., barcodes), semi-visible (e.g., Fujitsu's steganographic technique ${ }^{1}$ ), and non-visible (e.g., RFID) tags. The non-visible tags often have the advantage of operating remotely (i.e., without the tag occupying a large solid angle from the perspective of the scanner), but remain expensive to mass-produce. Visible tags such as two-dimensional barcodes, however, may be inexpensively printed on existing surfaces, but lack robustness at low solid angles from the scanning camera. In this work, we develop a printable tagging solution (“AugTag”) that may overcome this disadvantage, and operate over longer distances.

\section{METHODOLOGY}

Let us first consider the operation of traditional black-and-white (BW) codes. Typically, high contrast BW edges are used to align and identify the 2-D barcode. While it works well under lower-light conditions and is compatible with laser-based scanning devices, this technique breaks down for CCDs at low solid angles. Ideally, a printable tag would satisfy the following constraints: I. Tag should work at a distance (and therefore be machine-readable at $\sim 20 \times 20$ pixels); II. Tag should work in dark rooms; III. Tag should be able to cope with color distortion, if color is used; IV. Tag should be distinguishable from background; V. Tag should support a large number of configurations. Existing BW symbologies

International Symposium on Photoelectronic Detection and Imaging 2007: Image Processing, edited by Liwei Zhou, Proc. of SPIE Vol. 6623, 662304, (2008) · 0277-786X/08/\$18 · doi: 10.1117/12.791268 
(Semacode ${ }^{2}$, QR code ${ }^{3}$, etc.) meet criteria II-V, but not I.

Here we propose a new technique that is more robust at low angles by making the entire tag a high-contrast region. Such an encoding is not possible with 2 colors, since there are at most two 2-colorings of the square lattice. However, for 3-colored tags, a large number of encodings become possible while making the entire tag high-contrast. We may conveniently select red-green-blue (RGB) as these colors to maximize immunity to color distortion of different hardware. In particular, we propose the use of 3-colorings of a finite square lattice that are rotationally unique as a symbology. Consider, as an example, the 6x6 AugTag shown in Figure 1.

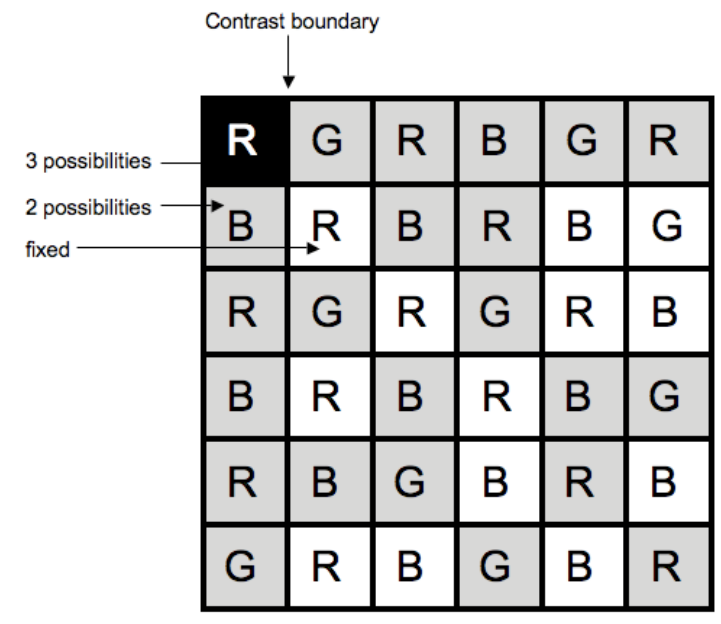

Figure 1: An example 3-coloring for a 6x6 AugTag. This encoding for a 6x6 AugTag is expected to represent approximately 24 bits of information. The number of possible colors for each cell allowed by the encoding, in order to maximize contrast, is indicated.

Our tag detection algorithm relies on maximizing the RGB contrast function,

$$
f_{x y}=\sqrt{\left(r_{x y}-g_{x y}\right)^{2}+\left(g_{x y}-b_{x y}\right)^{2}+\left(r_{x y}-b_{x y}\right)^{2}},
$$

where $r, g, b$ denote the image color channels, over a minimal square area $A$ as given by,

$$
\tilde{f}_{A}=\frac{1}{A^{3 / 4}} \iint_{A} f_{x y} d x d y .
$$

\section{DATA}

We performed preliminary tests of our 3-coloring 2D barcodes using a 0.3-megapixel camera, which would be a minimal baseline for a mobile phone, from full field down to $\sim 1 / 30$ of field. Some examples of the barcode detection are shown in Figure 2, with green circles indicating the associated square regions in the image field. 

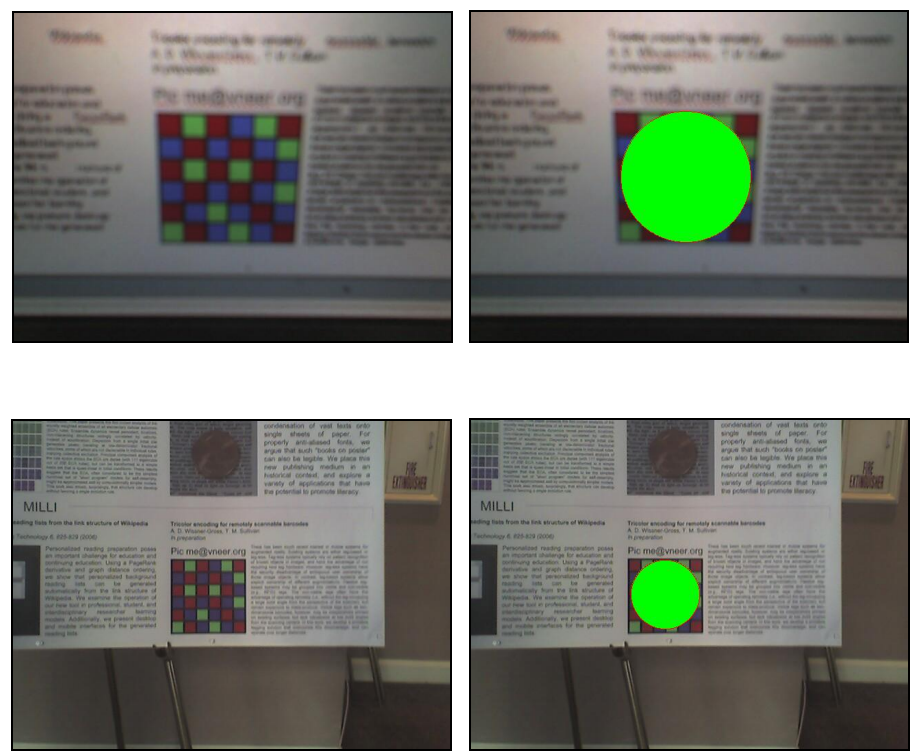

Figure 2: Detection of AugTags in 0.3-MP cameraphone images. Images before (left) and after (right) detection are shown, and image regions identified as containing the tags are inscribed with green circles by the software.

\section{RESULTS}

Our preliminary tests indicate that barcode detection is robust in the $>1 / 30$ image field range, which is a first step toward low solid angle detection and identification of the tags. Further work will be needed to investigate the sensitivity of actual tag identification, versus detection, to angle and lighting conditions.

\section{CONCLUSIONS}

Its diminutive size, ease of use and low production cost may eventually make the AugTag suitable for numerous practical applications. It lends itself especially well to use in product marketing. For example, retail apparel may be labeled or otherwise marked with AugTags, permitting users to identify the brand of clothing worn by another person and to see an image of the product used on a model in a retail store, perhaps with suggested coordinate garments. They may also provide detailed instructions, providing customized, part-by-part assembly instructions. In online apparel marketing, vendors may associate downloadable AugTags with individual garments that may then be printed, applied to one's person and photographed in a mirror. An augmented image returned to the potential customer will demonstrate how the apparel would appear on the consumer, thereby permitting a virtual changing room. Likewise, vendors of goods for use in the construction and design of residential and commercial spaces can offer customers AugTags to place in the area in which their product will sit. A photograph would then demonstrate to the customer how that product would look on site. For example, an AugTag related to a sofa could be placed on the wall against which the sofa will sit. A photograph returned from the AugTag server would then display an image of how the sofa would look in his or her room. Outside of the apparel industry, real estate agents can apply AugTags to the front of properties for sale or rent that visually open up the front of the property and allow potential customers to view the interior. 
By contrast, AugTags could be used to make dramatic augmentations to exterior realities. Historical commissions, museums, and private individuals may use them to demonstrate to users historical reconstructions of homes, buildings and entire street- and cityscapes. Contractors and architects can also apply images of AugTags to display underlying images of wiring and piping to permit non-destructive renovation, especially helpful in historic homes. Cities can also maintain the simplicity and clarity of street signage by using standard text and supplementing it with AugTags, which present detailed information about local resources and directions to commonly traveled local destinations. Advertisers on billboards can beautify the landscape, using AugTags to supplement attractive signage with helpful information about the store being advertised, including images of the store's interior. They may even be used by college students to leave notes, messages, and images on dormitory doors, in place of traditional chalk- and whiteboards.

In a similar vein, AugTags offer numerous ways to mark up the exterior of the human body, permitting promising medical applications. Physicians, nurses, and private individuals may use AugTags to apply x-rays and other images to patient bodies and garments for easily accessible visuals that are closely related to the patient's body. Similarly, they may be used on patient wristbands or identification for immediate and easily accessible information on the patients' allergies and other health concerns. Non-medical bodily applications include the creation of programmable tattoos and other body-marks.

\section{ACKNOWLEDGEMENTS}

A.W.-G. gratefully acknowledges financial support from the Fannie and John Hertz Foundation.

\section{REFERENCES}

1. G. J. Kewney, "Fujitsu to demonstrate 'invisible messages' for camera phone users at Technology Forum," Newswireless.net (posted January 24, 2006), http://www.newswireless.net/index.cfm/article/2583 .

2. Semacode SDK Technical Paper, Semacode Corporation, http://semacode.org/about/technical/ .

3. “About 2D Code,” Denso-Wave Incorporated, http://www.denso-wave.com/qrcode/aboutqr-e.html . 